\title{
Accelerating universe from the evolution of extra dimensions
}

\author{
Je-An Gu* and W.-Y. P. Hwang ${ }^{\dagger}$ \\ Department of Physics, National Taiwan University, Taipei 106, Taiwan, Republic of China
}

(Received 10 January 2002; published 28 June 2002)

\begin{abstract}
In this paper we propose that the accelerating expansion of the present matter-dominated universe, as suggested by the recent distance measurements of type Ia supernovae, is generated along with the evolution of space in extra dimensions. The Einstein equations are first analyzed qualitatively and then solved numerically, so as to exhibit explicitly these patterns of the accelerating expansion in this scenario. A fine-tuning problem associated with such a scenario is also described and discussed.
\end{abstract}

DOI: $10.1103 /$ PhysRevD.66.024003

PACS number(s): 04.50.+h

\section{INTRODUCTION}

The recent distance measurements of type Ia supernovae suggest an accelerating expansion of the present universe $[1,2]$. In many of the current cosmological models, the present accelerating expansion is driven by an energy source called "dark energy", with a positive cosmological constant [3] or the so-called "quintessence" (a slowly evolving scalar field $[4,5])$ as a possible candidate. Instead of attributing this acceleration to the mysterious dark energy, we consider in this paper the possible existence of extra spatial dimensions and explore the feasibility of producing the present accelerating expansion via the evolution of these extra dimensions.

The application of extra dimensions is a general feature in theories beyond the standard model, especially in theories for unifying gravity and other forces, such as superstring theory. These extra dimensions should be "hidden" for consistency with observations. Various scenarios for "hidden" extra dimensions have been proposed, for example, a brane world with large compact extra dimensions in factorizable geometry proposed by Arkani-Hamed et al. [6] (see also [7]), and a brane world with noncompact extra dimensions in nonfactorizable geometry proposed by Randall and Sundrum [8]. In this paper, we employ the simplest scenario: small compact extra dimensions in factorizable geometry, as introduced in the Kaluza-Klein theories [9].

We study spatially homogeneous, isotropic, perfect-fluid cosmological models in $(1+3+n)$ dimensions where $n$ is the number of extra dimensions. In Sec. II, we first obtain, from the Einstein equations, some general features of the evolution of the higher-dimensional space, especially for a radiation-dominated universe and a (nonrelativistic) matterdominated universe. In Sec. III, we then explore the possibility of producing an accelerating expansion of the ordinary three-space via the evolution of "extra space" for a matterdominated universe. We analyze the Einstein equations to show qualitatively the behavior of this evolution and obtain numerical solutions which illustrate explicitly the accelerating expansion of the ordinary three-space along with the collapse of the extra space. We note that, while the KaluzaKlein cosmology and inflation in higher-dimensional space-

\footnotetext{
*Email address: jagu@ phys.ntu.edu.tw

†Email address: wyhwang@phys.ntu.edu.tw
}

time in connection with the early universe were discussed widely in the 1980s [10,11] (for a review, see [12]; for recent work, see $[13,14])$, the focus of this paper has to do with the present accelerating matter-dominated universe.

\section{EVOLUTION OF SPACES IN ORDINARY THREE DIMENSIONS AND EXTRA DIMENSIONS: GENERAL FEATURES}

We consider a metric of a $(3+n+1)$-dimensional spacetime, in which both the ordinary three-space and the extra (or "hidden") space are homogeneous and isotropic:

$$
\begin{aligned}
d s^{2}= & d t^{2}-a^{2}(t)\left(\frac{d r_{a}^{2}}{1-k_{a} r_{a}^{2}}+r_{a}^{2} d \Omega_{a}^{2}\right) \\
& -b^{2}(t)\left(\frac{d r_{b}^{2}}{1-k_{b} r_{b}^{2}}+r_{b}^{2} d \Omega_{b}^{2}\right), \\
& 0 \leqslant r_{b} \leqslant 1
\end{aligned}
$$

where $a(t)$ and $b(t)$ are scale factors, and the values of $k_{a}$ and $k_{b}$ are related to the curvatures of the ordinary threespace and the extra space, respectively. Assuming that the matter content in this higher-dimensional space is taken to be a perfect fluid, we can write the Einstein equations, which govern the evolution of the ordinary three-space and the extra space, as

$$
\begin{gathered}
3\left[\left(\frac{\dot{a}}{a}\right)^{2}+\frac{k_{a}}{a^{2}}\right]+\frac{n(n-1)}{2}\left[\left(\frac{\dot{b}}{b}\right)^{2}+\frac{k_{b}}{b^{2}}\right] \\
+3 n \frac{\dot{a} \dot{b}}{a} \frac{\dot{b}}{b}=8 \pi \bar{G} \bar{\rho}, \\
2 \frac{\ddot{a}}{a}+n \frac{\ddot{b}}{b}+\left[\left(\frac{\dot{a}}{a}\right)^{2}+\frac{k_{a}}{a^{2}}\right]+\frac{n(n-1)}{2}\left[\left(\frac{\dot{b}}{b}\right)^{2}+\frac{k_{b}}{b^{2}}\right] \\
+2 n\left(\frac{\dot{a}}{a}\right)\left(\frac{\dot{b}}{b}\right)=-8 \pi \bar{G} \bar{p}_{a},
\end{gathered}
$$




$$
\begin{gathered}
3 \frac{\ddot{a}}{a}+(n-1) \frac{\ddot{b}}{b}+3\left[\left(\frac{\dot{a}}{a}\right)^{2}+\frac{k_{a}}{a^{2}}\right] \\
+\frac{(n-1)(n-2)}{2}\left[\left(\frac{\dot{b}}{b}\right)^{2}+\frac{k_{b}}{b^{2}}\right] \\
+3(n-1)\left(\frac{\dot{a}}{a}\right)\left(\frac{\dot{b}}{b}\right)=-8 \pi \bar{G} \bar{p}_{b},
\end{gathered}
$$

where $\bar{G}$ and $\bar{\rho}$ are the gravitational constant and the energy density in the higher-dimensional world, and $\bar{p}_{a}$ and $\bar{p}_{b}$ are the pressures in the ordinary three-space and the extra space, respectively. Assuming simple equations of state $\bar{p}_{a}=\omega_{a} \bar{\rho}_{a}$ and $\bar{p}_{b}=\omega_{b} \bar{\rho}_{b}$ with constant state parameters $\omega_{a}$ and $\omega_{b}$, the conservation of stress energy gives rise to

$$
\bar{\rho} \propto a^{-3\left(1+\omega_{a}\right)} b^{-n\left(1+\omega_{b}\right)} .
$$

In this paper we assume $k_{b}=0$ for simplicity and study the evolution of the scale factors $a(t)$ and $b(t)$ from Eqs. (2)-(4). First of all, we consider a radiation-dominated universe with

$$
\begin{aligned}
& \bar{p}_{a}=\frac{1}{3} \bar{\rho}, \\
& \bar{p}_{b}=0 .
\end{aligned}
$$

For such a universe, we can read off, from Eqs. (2)-(4), a solution with constant $b$ (static extra dimensions), as shown in the following. For constant $b$, Eqs. (2)-(4) become

$$
\begin{aligned}
\left(\frac{\dot{a}}{a}\right)^{2}+\frac{k_{a}}{a^{2}} & =\frac{8 \pi \bar{G}}{3} \bar{\rho}, \\
2 \frac{\ddot{a}}{a}+\left(\frac{\dot{a}}{a}\right)^{2}+\frac{k_{a}}{a^{2}} & =-\frac{8 \pi \bar{G}}{3} \bar{\rho}, \\
\frac{\ddot{a}}{a}+\left(\frac{\dot{a}}{a}\right)^{2}+\frac{k_{a}}{a^{2}} & =0 .
\end{aligned}
$$

As a consistency check, we note that Eq. (8) can be derived from Eqs. (6) and (7), the latter being the equations that describe the evolution of a four-dimensional radiationdominated universe in the standard cosmology. In addition, the constant $b$ solution is stable under small perturbations of scale factors $a(t)$ and $b(t)$, a fact that can be shown straightforwardly. In other words, we can retrieve the ordinary evolution path of a radiation-dominated universe within a higher-dimensional space-time with static extra dimensions. On the other hand, for a matter-dominated universe with $\bar{p}_{a}$ $=\bar{p}_{b}=0$, there is no solution corresponding to constant $b$ (unless $\bar{\rho}=0$ ).
As a parenthetical remark, if we wish to obtain a solution with the same evolution path as a four-dimensional matterdominated universe in the standard cosmology, that is, $b=$ const and $a(t)$ satisfies

$$
\begin{aligned}
\left(\frac{\dot{a}}{a}\right)^{2}+\frac{k_{a}}{a^{2}} & =\frac{8 \pi \bar{G}_{-}}{3}, \\
2 \frac{\ddot{a}}{a}+\left(\frac{\dot{a}}{a}\right)^{2}+\frac{k_{a}}{a^{2}} & =0,
\end{aligned}
$$

the matter in the extra space needs to provide negative pressure

$$
\bar{p}_{b}=-\frac{1}{2} \bar{\rho}
$$

although it provides negligible pressure in the ordinary threespace. It seems unusual to provide pressure in such a strange manner. Nevertheless, it is in fact still possible that, if the nonrelativistic "particles" in the ordinary three-space are in fact extended objects (like strings) in the extra space, they may provide pressure in this manner.

Assuming $k_{a}=k_{b}=0$ for simplicity and using the simple equations of state $\bar{p}_{a}=\omega_{a} \bar{\rho}$ and $\bar{p}_{b}=\omega_{b} \bar{\rho}$ with constant $\omega_{a}$ and $\omega_{b}$, we obtain

$$
\begin{aligned}
\alpha(t) \equiv & \left(1-3 \omega_{a}+2 \omega_{b}\right) \frac{\dot{a}}{a} \\
& -\left[1+(n-1) \omega_{a}-n \omega_{b}\right] \frac{\dot{b}}{b} \\
\propto & \frac{1}{a^{3} b^{n}} \propto \frac{1}{V_{3+n}},
\end{aligned}
$$

where $\alpha(t)$ in many cases characterizes roughly the difference between the expansion rates of the ordinary three-space and the extra space, and $V_{3+n}$ is the volume of the $(3+n)$-dimensional space. It follows from Eq. (11) that the difference $\alpha(t)$ will either decrease or grow as the volume $V_{3+n}$ of the higher-dimensional world grows or decreases. For a radiation-dominated universe with $\bar{p}_{a}=\frac{1}{3} \bar{\rho}$ and $\bar{p}_{b}=0$, we have

$$
\alpha(t)=\frac{n+2}{3} \frac{\dot{b}}{b} \propto \frac{1}{a^{3} b^{n}} \propto \frac{1}{V_{3+n}} .
$$

Accordingly, if $V_{3+n} \quad$ [the volume of overall $(3+n)$-dimensional space] is growing, the expansion rate of the extra space will drop and approach zero. We note that this feature also indicates the stability of the constant- $b$ solution mentioned above in a radiation-dominated universe. On the other hand, for a matter-dominated universe with $\bar{p}_{a}$ $=\bar{p}_{b}=0$ or for a more general case with $\omega_{a}=\omega_{b} \neq 1 / 3$, we have 


$$
\alpha(t) \propto \frac{\dot{a}}{a}-\frac{\dot{b}}{b} \propto \frac{1}{a^{3} b^{n}} \propto \frac{1}{V_{3+n}} .
$$

Accordingly, if the volume $V_{3+n}$ is growing, the expansion rates of the ordinary three-space and the extra space will tend to approach each other. If the volume $V_{3+n}$ is decreasing, on the other hand, with one expanding space and one collapsing space, then either $|\dot{a} / a|$ or $|\dot{b} / b|$ will become larger and larger. We note that an increasing positive expansion rate represents an accelerating expansion. Thus, the accelerating expansion of our universe (the ordinary three-space) may be generated along with the collapse of the extra space, as will be discussed in the next section.

\section{ACCELERATING EXPANSION OF THE ORDINARY THREE-SPACE GENERATED BY THE EVOLUTION OF EXTRA DIMENSIONS}

In this section, we consider a $(1+3+n)$-dimensional matter-dominated universe and wish to explore, both analytically and numerically, the possibility of generating the accelerating expansion of the ordinary three-space via the the evolution of the extra space. Assuming $k_{a}=k_{b}=0$ for simplicity and using the equations of states $\bar{p}_{a}=\bar{p}_{b}=0$, we can rearrange Eqs. (3) and (4) to become

$$
\begin{gathered}
(n+2) \frac{\ddot{a}}{a}+(2 n+1)\left(\frac{\dot{a}}{a}\right)^{2}+n(n-1) \frac{\dot{a}}{a} \frac{\dot{b}}{b} \\
-\frac{n(n-1)}{2}\left(\frac{\dot{b}}{b}\right)^{2}=0, \\
(n+2) \frac{\ddot{b}}{b}-3\left(\frac{\dot{a}}{a}\right)^{2}+6 \frac{\dot{a}}{a} \frac{\dot{b}}{b}+\frac{(n-1)(n+4)}{2}\left(\frac{\dot{b}}{b}\right)^{2}=0,
\end{gathered}
$$

or, equivalently, with $u$ and $v$ defined as $u(t) \equiv \dot{a} / a$ and $v(t) \equiv \dot{b} / b$,

$$
\begin{gathered}
(n+2) \dot{u}+3(n+1) u^{2}+n(n-1) u v \\
-\frac{n(n-1)}{2} v^{2}=0, \\
(n+2) \dot{v}-3 u^{2}+6 u v+\frac{n(n+5)}{2} v^{2}=0 .
\end{gathered}
$$

From Eq. (14), we can read off the condition for $\ddot{a} / a>0$ (accelerating ordinary three-space),

$$
\frac{\dot{b}}{b}>\left[1+\sqrt{\frac{(n+1)(n+2)}{n(n-1)}}\right] \frac{\dot{a}}{a} \equiv J_{+}\left(\frac{\dot{a}}{a}\right),
$$

or

$$
\frac{\dot{b}}{b}<\left[1-\sqrt{\frac{(n+1)(n+2)}{n(n-1)}}\right] \frac{\dot{a}}{a} \equiv J_{-}\left(\frac{\dot{a}}{a}\right),
$$

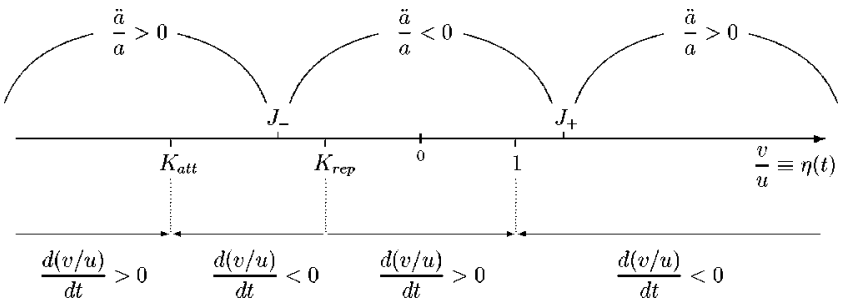

FIG. 1. An illustration of (1) the situations for various acceleration states of the ordinary three-space and (2) evolution paths of the fraction $\eta=v / u$.

and also the condition for $\ddot{a} / a<0$ (decelerating ordinary three-space),

$$
J_{-}\left(\frac{\dot{a}}{a}\right)<\frac{\dot{b}}{b}<J_{+}\left(\frac{\dot{a}}{a}\right) .
$$

We can see that the fraction $\eta(t)[\equiv v(t) / u(t)]$ is the key quantity in the above conditions for swaying the acceleration status of the ordinary three-space. Therefore it is essential to investigate possible evolution paths of the fraction $\eta(t)$, i.e., to see the behavior of $d \eta / d t$ in different regions of $\eta$.

Using Eqs. (16) and (17), we obtain the conditions

$$
\begin{aligned}
& \frac{d \eta}{d t}>0 \quad \text { for } \quad \eta<K_{a t t} \quad \text { or } \quad K_{r e p}<\eta<1, \\
& \frac{d \eta}{d t}<0 \quad \text { for } \quad K_{a t t}<\eta<K_{\text {rep }} \quad \text { or } \quad \eta>1,
\end{aligned}
$$

where $K_{a t t}$ and $K_{\text {rep }}$ are defined by

$$
\begin{gathered}
K_{a t t}=-\frac{3+\sqrt{3(n+2) / n}}{n-1}, \\
K_{r e p}=-\frac{3-\sqrt{3(n+2) / n}}{n-1} .
\end{gathered}
$$

We note that the subscripts att and rep denote "attractor" and "repeller," respectively. Their meanings will be described in the following discussion.

The conditions in Eqs. (18)-(22) can be summarized in the "flow diagram" Fig. 1. The upper part of Fig. 1 illustrates the conditions in Eqs. (18)-(20) for the behavior of $\ddot{a} / a$, while the lower part illustrates the conditions in Eqs. (21) and (22) for the behavior of $d \eta / d t$. We note that $J_{+}$, $J_{-}, K_{a t t}$, and $K_{\text {rep }}$ always obey the order

$$
K_{a t t}<J_{-}<K_{r e p}<0<1<J_{+}
$$

for $n \geqslant 2$. In Fig. 1, we can see that there are two "attractors" at $\eta=K_{\text {att }}$ and at $\eta=1$, and one "repeller" at $\eta=K_{\text {rep }}$. The higher-dimensional world will approach the state at $\eta$ $=K_{a t t}$ for the class of initial conditions satisfying $\eta$ $<K_{\text {rep }}$, while it will approach $\eta=1$ for the other initialcondition class $\eta>K_{\text {rep }}$. Accordingly, the higher- 

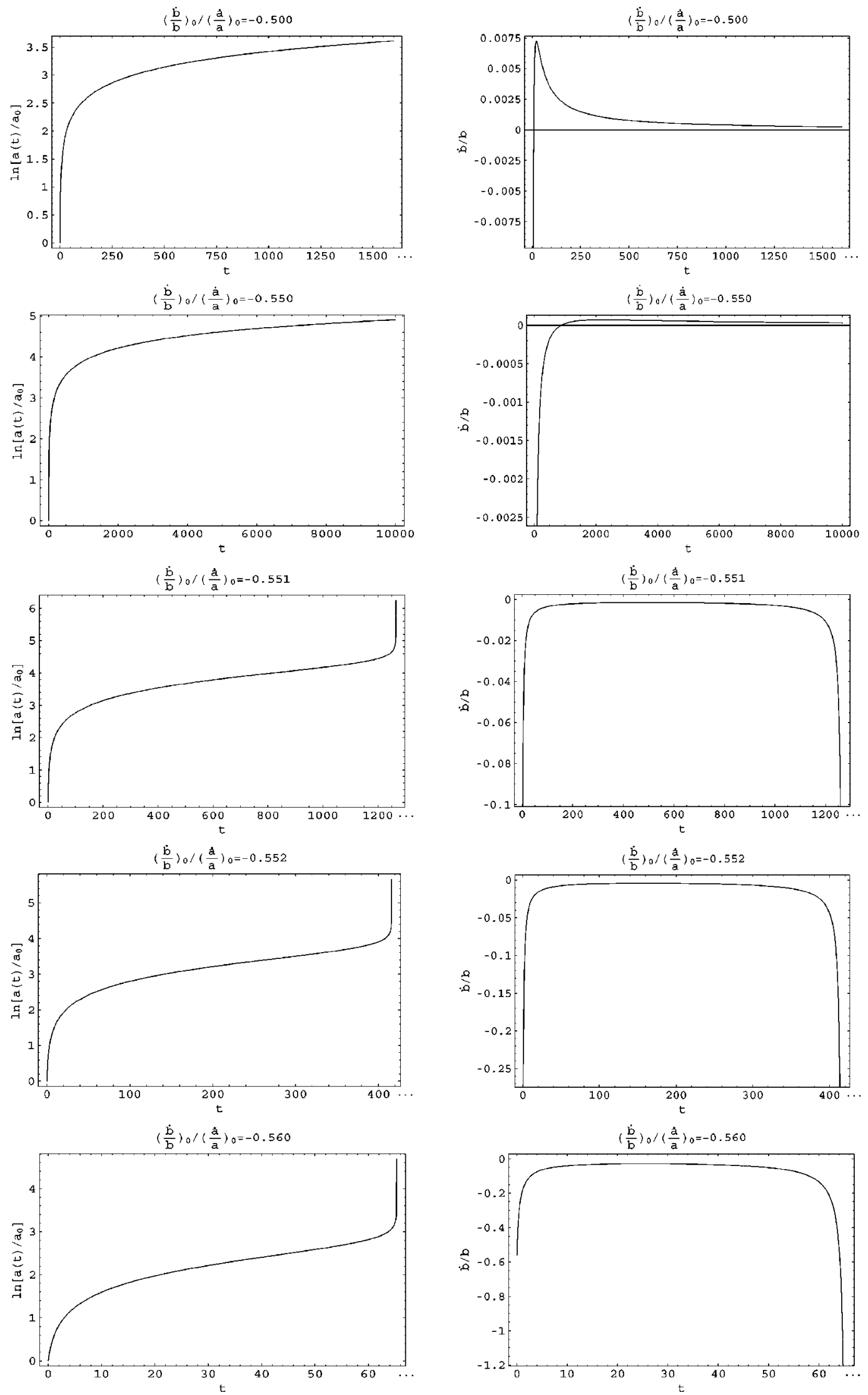

FIG. 2. The evolution paths-plots of $\ln \left[a(t) / a_{0}\right]$ and $\dot{b} / b$. The quantities $\dot{b} / b$ and $t$ are in units of $(\dot{a} / a)_{0}$ and $(\dot{a} / a)_{0}^{-1}$, respectively, where the subscript 0 denotes the initial time $t_{0}=t_{E Q}$ (which is set to be zero). 


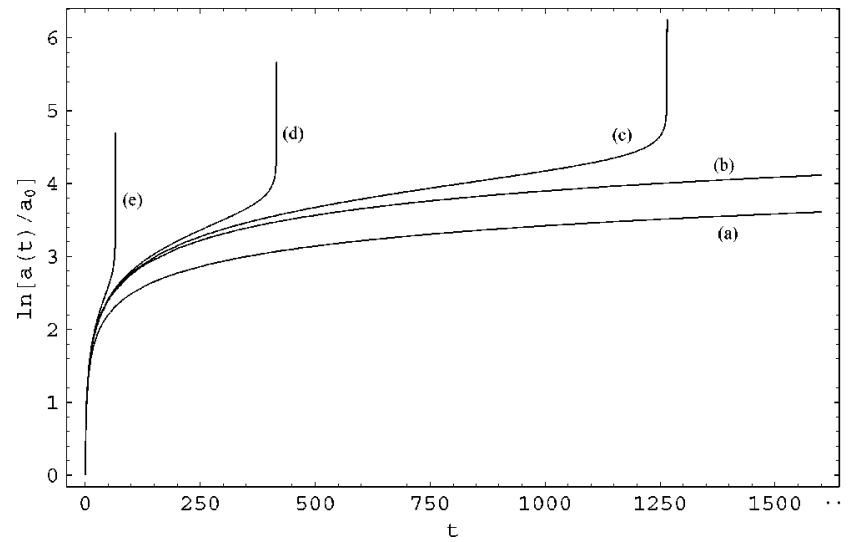

FIG. 3. The evolution paths-plot of $\ln \left[a(t) / a_{0}\right]$ : (a) $(\dot{b} / b)_{0}=$ $-0.500(\dot{a} / a)_{0}, \quad$ (b) $\quad(\dot{b} / b)_{0}=-0.550(\dot{a} / a)_{0}, \quad$ (c) $\quad(\dot{b} / b)_{0}=$ $-0.551(\dot{a} / a)_{0}, \quad(\mathrm{~d}) \quad(\dot{b} / b)_{0}=-0.552(\dot{a} / a)_{0}, \quad(\mathrm{e}) \quad(\dot{b} / b)_{0}=$ $-0.560(\dot{a} / a)_{0}$. The time $t$ is in units of $(\dot{a} / a)_{0}^{-1}$, where the subscript 0 denotes the initial time $t_{0}=t_{E Q}$ (which is set to be zero).

dimensional world possesses four kinds of evolution path corresponding to different $\eta_{0}$ (the initial value of $\eta$ ):

$\eta_{0}>J_{+}, \quad$ accelerate first and then decelerate,

$$
K_{\text {rep }}<\eta_{0}<J_{+}, \quad \text { always decelerate, }
$$

$J_{-}<\eta_{0}<K_{\text {rep }}, \quad$ decelerate first and then accelerate,

$$
\eta_{0}<J_{-}, \quad \text { always accelerate. }
$$

Consequently, we can see that the situation in Eq. (26c) may properly describe our universe. In this situation, the universe is in the region $\left(J_{-}, K_{r e p}\right)$ initially and the ordinary threespace expands in a decelerating manner during the period when $\eta$ evolves within this region, while the extra space always collapses. After some time the ordinary three-space will start to accelerate as $\eta$ passes $J_{-}$in the collapsing process of the extra space. (Please see the Note added.)

For a concrete illustration, we analyze Eqs. (14) and (15) numerically for the case of $n=2$. We consider the initial time to be the moment when the nonrelativistic matter starts to take over and dominate the universe, which is usually denoted by $t_{E Q}$ (where $E Q$ means "matter-radiation equality"). The results are demonstrated in Fig. 2 and Fig. 3, in which five evolution paths corresponding to various initial conditions are drawn. The five initial conditions we employ are (a) $(\dot{b} / b)_{0}=-0.500(\dot{a} / a)_{0}$, (b) $(\dot{b} / b)_{0}=-0.550(\dot{a} / a)_{0}$, (c) $(\dot{b} / b)_{0}=-0.551(\dot{a} / a)_{0},(\mathrm{~d})(\dot{b} / b)_{0}=-0.552(\dot{a} / a)_{0}$, and (e) $(\dot{b} / b)_{0}=-0.560(\dot{a} / a)_{0}$. The plots in the left column of Fig. 2 sketch $\ln \left[a(t) / a_{0}\right]$, and the plots in the right column sketch $\dot{b}(t) / b(t)$. Figure 3 combines the plots in the left column of Fig. 2 for comparison. The value of $\dot{b} / b$ and the time $t$ are in units of $(\dot{a} / a)_{0}$ and $(\dot{a} / a)_{0}^{-1}$ (the Hubble time at $t_{0}=t_{E Q}$, which is set to be zero here), respectively. We can see that the evolution path is very sensitive to the initial value of $\eta$ and, more importantly, there exists a critical value $\eta_{c r}$, which is indeed the parameter $K_{\text {rep }}$ and is about -0.55051 for $n=2$, such that the ordinary three-space will either expand in a decelerating manner for $\eta_{0}>\eta_{c r}$ or accelerate eventually for $\eta_{0}<\eta_{c r}$.

To sum up, the analysis of the matter-dominated case with $k_{a}=k_{b}=0$ leads to Fig. 1. Four classes of evolution path, as described in Eqs. (26a)-(26d), can be read off from Fig. 1. The situation that may appropriately describe our universe is the one suggested by Eq. (26c), in which the ordinary threespace decelerates first and then accelerates along with the collapse of the extra space. Figures 2 and 3 show five evolution paths corresponding to five different initial conditions, where paths (a) and (b) illustrate the situation in Eq. (26b) and paths (c)-(e) illustrate the situation in Eq. (26c). There exists a critical value $\eta_{c r}$, which is exactly the parameter $K_{\text {rep }}$, dividing these two classes of evolution path. For the case with $\eta_{0}<\eta_{c r}$ corresponding to the situation in Eq. (26c), the period before the acceleration starts is sensitive to the the initial value of $\eta, \eta_{0}$. If we require the expansion of our universe to decelerate for a long enough period before the acceleration starts in order to conserve the concordance between observations and theories regarding the early universe, the initial value of $\eta$ has to be chosen in a delicate way such that it is close enough to $\eta_{c r}$. Therefore this scenario so far has a fine-tuning problem.

\section{DISCUSSION AND SUMMARY}

We have investigated the scenario of producing the accelerating expansion of the present universe via evolving small extra dimensions. For a radiation-dominated universe, such as our early universe, we obtain a stable solution with static extra dimensions. Accordingly, the existence of extra dimensions may have no significant influence on the evolution of the ordinary three-space. This is a good feature which we need for preserving the concordance between observations and current theories regarding the early (radiationdominated) universe, especially for primordial nucleosynthesis. On the contrary, such a solution with static extra dimensions does not exist for the present matter-dominated universe.

The features of the evolution can also be read off from Eq. (11), or Eqs. (12) and (13), which are derived from Eq. (11). Equation (12) shows the decreasing expansion rate of the extra space along with the increase of the $(3+n)$-dimensional volume $V_{3+n}$. This implies the stability of the solution with static extra dimensions in the radiationdominated universe for the case of $k_{a}=k_{b}=0$ as already mentioned above. On the other hand, Eq. (13) shows two possible evolution patterns of the matter-dominated universe: (i) The expansion rates of the ordinary three-space and the extra space tend to catch up with each other along with the increase of the $(3+n)$-dimensional volume $V_{3+n}$. (ii) One of these two expansion rates is positive and increasing, while the other is negative and decreasing, along with the decrease of the $(3+n)$-dimensional volume $V_{3+n}$. We note that an increasing positive expansion rate represents an accelerating expansion.

A quantitative analysis of the matter-dominated case with 
$k_{a}=k_{b}=0$ leads to Fig. 1, which indicates four classes of evolution path. A universe that decelerates first and then accelerates is included in one of them. Therefore the accelerating expansion of the present universe may be appropriately described in this scenario. In addition, the case with two extra dimensions is analyzed in detail. The five resultant evolution paths we draw demonstrate the existence of a critical value for the initial condition $\eta_{0}$, which divides two classes of path: the one in which the universe decelerates first and then accelerates and the other in which the universe always decelerates. We note that the critical value $\eta_{c r}$ is exactly the parameter $K_{\text {rep }}$, a "repeller" in the flow diagram. However, the existence of the critical value (or the "repeller") also implies a fine-tuning problem, i.e., the initial value of $\eta$ has to be chosen delicately so that it is close enough to the critical value $\eta_{c r}$ in order to possess a long enough decelerating epoch followed by an accelerating epoch.

The existence of extra dimensions is a general feature in theories beyond the standard model in particle physics. It may manifest itself as a source of energy in the ordinary three-space, such as "effective" dark energy or even "effec- tive" dark matter. The geometrical structure and the evolution pattern of extra dimensions therefore may play an important role in cosmology. In this work we study a simple scenario of extra dimensions that is subject to a fine-tuning problem. Nevertheless, other scenarios with richer structures, such as those in [6] and [8], may also provide suitable evolution patterns and are worthy of being further investigated.

Note added. For the sake of simplicity, we have in this paper considered only the case with $k_{a}=k_{b}=0$, i.e., both our ordinary three-space and the extra space are flat. The general situations with nonzero $k_{a}$ or $k_{b}$ clearly may offer many interesting possibilities and are currently under serious investigation.

\section{ACKNOWLEDGMENTS}

One of us (J.-A.G.) wishes to thank Professor W. F. Kao for helpful discussions. This work was supported in part by the National Science Council, Taiwan, Republic of China (NSC 90-2112-M-002-028) and by the CosPA project of the Ministry of Education (MOE 89-N-FA01-1-4-3).
[1] Supernova Cosmology Project Collaboration, S. Perlmutter et al., Astrophys. J. 517, 565 (1999).

[2] Supernova Search Team Collaboration, A.G. Riess et al., Astron. J. 116, 1009 (1998).

[3] L.M. Krauss and M.S. Turner, Gen. Relativ. Gravit. 27, 1137 (1995); J.P. Ostriker and P.J. Steinhardt, Nature (London) 377, 600 (1995); A.R. Liddle, D.H. Lyth, P.T. Viana, and M. White, Mon. Not. R. Astron. Soc. 282, 281 (1996).

[4] R.R. Caldwell, R. Dave, and P.J. Steinhardt, Phys. Rev. Lett. 80, 1582 (1998).

[5] Je-An Gu and W.-Y.P. Hwang, Phys. Lett. B 517, 1 (2001); L.A. Boyle, R.R. Caldwell, and M. Kamionkowski, astro-ph/0105318.

[6] N. Arkani-Hamed, S. Dimopoulos, and G. Dvali, Phys. Lett. B 429, 263 (1998); I. Antoniadis, N. Arkani-Hamed, S. Dimopoulos, and G.R. Dvali, ibid. 436, 257 (1998).
[7] I. Antoniadis, Phys. Lett. B 246, 377 (1990).

[8] L. Randall and R. Sundrum, Phys. Rev. Lett. 83, 3370 (1999); 83, 4690 (1999).

[9] T. Kaluza, Sitzungsber. K. Preuss. Akad. Wiss. K1, 966 (1921); O. Klein, Z. Phys. 37, 895 (1926) [Surv. High Energy Phys. 5, 241 (1926)].

[10] P.G. Freund, Nucl. Phys. B209, 146 (1982).

[11] E. Alvarez and M. Belen Gavela, Phys. Rev. Lett. 51, 931 (1983); D. Sahdev, Phys. Lett. 137B, 155 (1984); E.W. Kolb, D. Lindley, and D. Seckel, Phys. Rev. D 30, 1205 (1984); R.B. Abbott, S.M. Barr, and S.D. Ellis, ibid. 30, 720 (1984).

[12] E.W. Kolb and M.S. Turner, The Early Universe (AddisonWesley, Redwood City, CA, 1998), and references therein.

[13] N. Arkani-Hamed, S. Dimopoulos, N. Kaloper, and J. MarchRussell, Nucl. Phys. B567, 189 (2000).

[14] T.R. Mongan, Gen. Relativ. Gravit. 33, 1415 (2001). 